${ }^{20}$ E. P. Lippincott and A. M. Bernstein, Phys. Rev.
$\underline{163}, 1170(1967)$.

\title{
Induced Decay of the Neutral Vacuum in Overcritical Fields Occurring in Heavy-Ion Collisions*
}

\author{
K. Smith, $\uparrow$ H. Peitz, B. Muller, and W. Greiner \\ Institut für Theoretische Physik der Universität Frankfurt, Frankfurt am Main, Germany
}

(Received 17 December 1973)

\begin{abstract}
In critical or nearly critical heavy-ion collisions, induced as well as spontaneous energyless $e^{-} e^{+}$pair creation result in the decay of the neutral vacuum. Induced transitions from the negative-energy continuum into a vacant molecular $1 s$ level can occur even in the absence of diving and produce a substantial enhancement and broadening of the previously considered spontaneous positron spectrum. Total cross sections of $5 \mathrm{~b}$ have been calculated for U-U collisions.
\end{abstract}

Intermediate quasimolecules, which will be formed in the collision of very heavy nuclei, show with respect to their electronic structure all properties of superheavy atoms. ${ }^{1-5}$ In the case that the charge number $Z_{1}+Z_{2}$ of the colliding nuclei is larger than the critical charge number $Z_{\mathrm{cr}} \simeq 169-172$, the $1 s_{1 / 2}$ level enters the negative-energy continuum. If the $K$ shell is ionized, $e^{+} e^{-}$creation results. This has to be interpreted as the decay of the neutral vacuum, which is unstable in overcritical fields. ${ }^{4}$

Up to now calculations were done for the spontaneous positron creation. ${ }^{6}$ The cross sections will be increased, however, by two effects because of the nonadiabacticity of the heavy-ion collision: First, during the "diving" process $e^{+} e^{-}$pairs are created, in addition to the spontaneous ones, by induced autoionization. Second, before and after the diving a large number of positrons will be created by induced transitions from the negative-energy continuum to the $1 s_{1 / 2}$ level. The latter transitions will occur even if there is no level-diving during the collision.

To calculate the total transition amplitude, all the transitions, from the negative-energy continuum to the $1 s_{1 / 2}$ level, along the classical ion path must be added coherently. ${ }^{7}$ These transitions may be approximately grouped into first, pre- and after-diving amplitudes $C_{P A, E}$, and second, the duringdiving amplitude $C_{D}$. Then we have

$$
\begin{aligned}
C_{P A, E}=\int_{-\infty}^{-t} \mathrm{cr} d t^{\prime} \exp \left\{(i / \hbar) \int_{-\infty}^{t^{\prime}} d t^{\prime \prime}\left[E-E_{1 s_{1 / 2}}\left(t^{\prime \prime}\right)\right]-(2 \hbar)^{-1} \int_{-\infty}^{t^{\prime}} \Gamma\left(t^{\prime \prime}\right) d t^{\prime \prime} M_{E}\left(t^{\prime}\right)\right\} \\
\quad+\int_{t_{\mathrm{cr}}}^{\infty} d t^{\prime} \exp \left\{(i / \hbar) \int_{-\infty}^{t^{\prime}} d t^{\prime \prime}\left[E-E_{1 s_{1 / 2}}\left(t^{\prime \prime}\right)\right]-(2 \hbar)^{-1} \int_{-\infty}^{t^{\prime}} \Gamma\left(t^{\prime \prime}\right) d t^{\prime \prime} M_{E}\left(t^{\prime}\right)\right\},
\end{aligned}
$$

where

$$
M_{E}\left(t^{\prime}\right)=-\left\langle\psi_{E}\left(t^{\prime}\right)\left|\left(\partial / \partial t^{\prime}\right)\right| \varphi\left(t^{\prime}\right)\right\rangle
$$

and $\Gamma\left(t^{\prime \prime}\right)$ represents the decay $1 s_{1 / 2}$ level due to the interaction with the continuum (to be determined later) and $t_{c r}$ denotes the "critical" time at which diving occurs. $\left|\psi_{E}\right\rangle$ and $|\varphi\rangle$ are the wave functions for the positron (negative-energy continuum) and the $1 s_{1 / 2}$ vacancy, respectively. The time integration can be replaced by an integration over $d R / v_{R}$ along the ion hyperbola, where $R$ is the distance of the two ions and $v_{R}$ the radial velocity.

The matrix element $M_{E}\left(t^{\prime}\right)$ can be computed by expanding the bound state $\left|\varphi\left(t^{\prime}\right)\right\rangle=|\varphi(R)\rangle$ and the continuum states $\left|\psi_{E}\left(t^{\prime}\right)\right\rangle=\left|\psi_{E}(R)\right\rangle$, which are eigenstates of the Hamiltonian $H(R)$, about the $1 s_{1 / 2}$ diving radius $R_{\text {cr }}$; i.e.,

$$
|\varphi(R)\rangle=f(R)\left\{\left|\varphi_{\mathbf{c r}}\right\rangle+\int d E^{\prime} g_{E^{\prime}}(R)\left|\psi_{E^{\prime}, \mathbf{c r}}\right\rangle\right\}, \quad\left|\psi_{E}(R)\right\rangle=a(E, R)\left\{\left|\varphi_{\mathbf{c r}}\right\rangle+\int d E^{\prime} b_{E^{\prime}}(E, R)\left|\psi_{E^{\prime}, \mathrm{cr}}\right\rangle\right\}
$$

with the following normalizations:

$$
\langle\varphi(R) \mid \varphi(R)\rangle=1, \quad\left\langle\psi_{E}(R) \mid \psi_{E^{\prime}}(R)\right\rangle=\delta\left(E-E^{\prime}\right), \quad\left\langle\psi_{E}(R) \mid \varphi(R)\right\rangle=0 .
$$

In the expression (3), the higher bound states, i.e., $2 p_{1 / 2}, 2 s_{1 / 2}$, etc., have been neglected. Such effects are expected to be most important at large ion separations where the matrix element $M_{E}(t)$ is 
small.

The matrix element (2) can now be written in terms of the expansion coefficients and reduces by use of Eq. (4) to

$$
M=\left\{a^{*}(E, R) f(R) \int d E^{\prime} b_{E^{\prime}} *(E, R)\left[(\partial / \partial R) g_{E^{\prime}}(R)\right]\right\} v_{R} .
$$

To solve for the expansion coefficients, the following matrix elements are needed:

$$
\epsilon(R)=\left\langle\varphi_{\mathrm{cr}}|V(R)| \varphi_{\mathrm{cr}}\right\rangle, \quad V_{E}(R)=\left\langle\psi_{E, \mathrm{cr}}|V(R)| \varphi_{\mathrm{cr}}\right\rangle \approx[\gamma(E) / 2 \pi]^{1 / 2} \epsilon(R), \quad V_{E E^{\prime}}(R)=\left\langle\psi_{E, \mathrm{cr}}|V(R)| \psi_{E^{\prime}, \mathrm{cr}}\right\rangle,
$$

where $V(R) \equiv H(R)-H\left(R_{\mathrm{cr}}\right)$.

When the small contributions from the continuum-continuum matrix elements $V_{E E^{\prime}}(R)$ are neglected the set of coupled-channel equations may be easily solved ${ }^{7}$ for the expansion coefficients. Upon substituting these coefficients into Eq. (5), the matrix element $M_{E}(t)$ may be readily computed. Once this matrix element is specified, the contribution from induced transitions to the total transition probability may be approximated by [Eq. (1)]

$$
W_{P A}\left(E_{p}, E_{I}, \theta\right)=\left|C_{F A}\right|^{2} d E_{p},
$$

where $E_{p}$ is the positron energy. The dependence of this probability on the ion energy $E_{I}$ and the scattering angle $\theta$ enters through the time integrations over the Rutherford trajectory.

The during-diving amplitude $C_{D}$ is given by ${ }^{7}$

$$
C_{D}=\frac{i}{\hbar} \int_{-t_{\mathrm{Cr}}}^{t_{\mathrm{Cr}}} d t V_{E}(t) \exp \left[\frac{i}{\hbar} \int_{-\infty}^{t} d t^{\prime}\left[E-E_{1 s_{1 / 2}}\left(t^{\prime}\right)\right]-\frac{1}{2 h} \int_{-\infty}^{t} \Gamma\left(t^{\prime}\right) d t^{\prime}\right],
$$

with the corresponding diving probability

$$
W_{D}\left(E_{p}, E_{I}, \theta\right)=\left|C_{D}\right|^{2} d E_{p} \text {. }
$$

The total probability for producing a positron with an energy between $E_{p}$ and $E_{p}+d E_{p}$ is then

$$
W_{T}\left(E_{p}, E_{I}, \theta\right)=\left|C_{P A}+C_{D}\right|^{2} d E_{p} \text {. }
$$

To compute these probabilities, the functions $\gamma(E)$ and $\epsilon(R)$ must be specified. The $\gamma(E)$ dependence was extracted from the resonance in the one-center continuum wave functions. ${ }^{4}$ While the resonance location $\epsilon(R)$ and the width $\Gamma_{E}(R)$ $=2 \pi\left|V_{E}(R)\right|^{2}$ depend on both the charge $Z$ and the separation $R$, as seen in Fig. 1, the ratio $\gamma(E)$ $=M_{e} c^{2} \Gamma_{E}(R) / \epsilon^{2}(R)$ depends only on energy to a good approximation. We used the approximation $\gamma(E)=\gamma_{0}\left(E-E_{0}\right)^{2} \exp \left[-\alpha\left(E-E_{0}\right)\right]$, where $\gamma_{0}, E_{0}$, and $\alpha$ are parameters in the present calculations. While this energy dependence is based on onecenter calculations, we assume that $\gamma(E)$ does not change appreciably when two-center wave functions are used. The large width of $\gamma(E)$ enables one to use $\Gamma(R)=\bar{\gamma}_{0} \epsilon^{2}(R)$ in the calculation of the line broadening [Eq. (1)]. The remaining function to be determined, $\epsilon(R)$, was extracted from the two-center Dirac-model ${ }^{8}$ eigenvalues.

The quantities $W_{P A}\left(E_{p}, \theta\right)$ and $W_{D}\left(E_{p}, \theta\right)$ are shown for the U-U system in Fig. 2(a). They are related to the cross section for positron production by

$$
\frac{d \sigma}{d E_{p} d \Omega_{\text {ion }}}=\frac{d \sigma_{R}}{d \Omega_{\text {ion }}} L_{0} W\left(E_{p}, \theta\right),
$$

where $d \sigma_{\mathrm{R}}$ is the differential Rutherford cross section and $L_{0}$ the initial $K$-hole probability, which has been taken in all our calculations to be $L_{0}=10^{-2}$ (see Ref. 7).

Figure 2(b) shows the total ionization probability $W_{T}\left(E_{p}, \theta\right)$. The solid curves demonstrate that with decreasing ion energy $E_{l}$, the energies $\bar{E}_{p}$ for the maximal positron cross sections are shifted to smaller values. This possibly allows to some extent a spectroscopy of the diving mechanism. For fixed ion energy and varying scattering angle $\theta$, the energy maximum is only slightly shifted, as can be seen from the dashed curves.

The positron production cross section in the en-

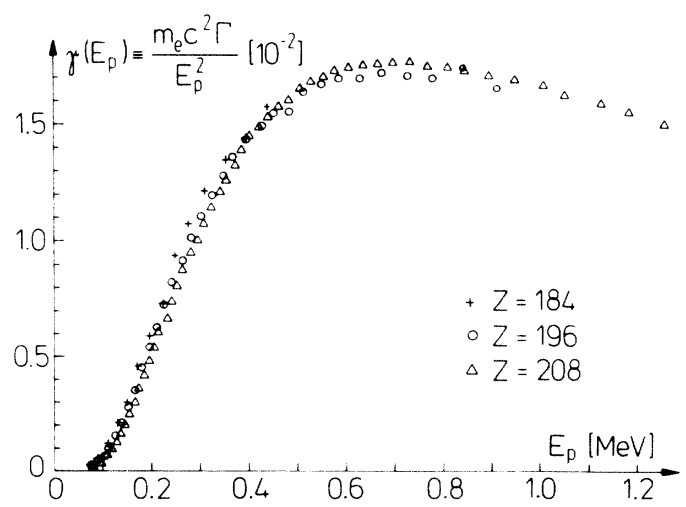

FIG. 1. The function $\gamma\left(E_{p}\right)$ for $180 \leqslant Z \leqslant 210$ for distances greater than $15 \mathrm{fm}$ (i.e., below the Coulomb barrier). Obviously, $\gamma\left(E_{p}\right)$ does not depend on $Z$ and $R$. 


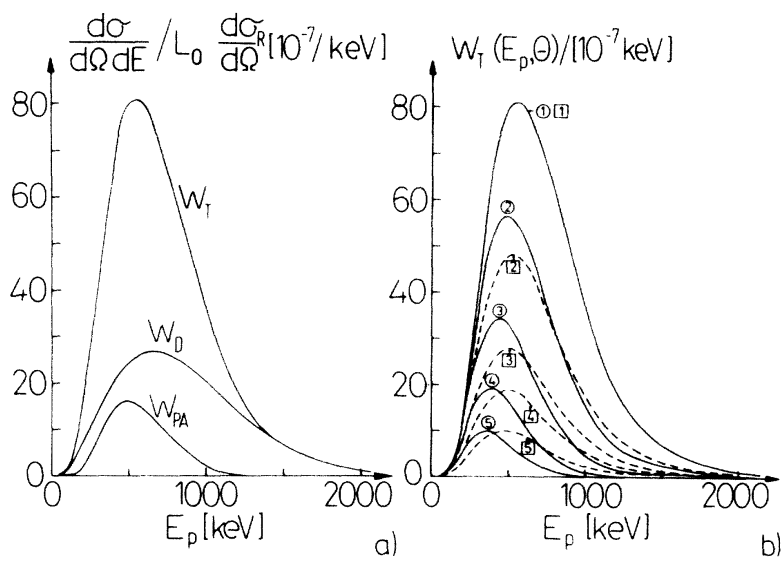

FIG. 2. (a) The probabilities $W_{P A}\left(E_{p}, \theta\right), W_{D}\left(E_{p}, \theta\right)$ and $W_{T}\left(E_{p}, \theta\right)$ for the system $U+U$ in the case of central collision with $E_{I}=812.5 \mathrm{MeV}$. (b) The solid lines show $W_{T}\left(E_{p}, \theta\right)$ at $\theta=180^{\circ}$ for the system $\mathrm{U} \rightarrow \mathrm{U}$ for various values of $R_{\min }$ (distance of closest approach in fermis), corresponding to different ion energies (given in parentheses, in MeV): (1) 15 (815.5), (2) 20 (609.4), (3) $25(478.5),(4) 30(406.3)$, (5) $35(348.2)$. In the last case $W_{T}\left(E_{p}, \theta\right)=W_{P A}\left(E_{p}, \theta\right)$ (no diving). The dashed curves show, for fixed ion energy $E_{I}=815.5 \mathrm{MeV}$, the change with $\theta$ : (1) $\theta=180^{\circ}$, (2) $\theta=75^{\circ}$, (3) $\theta=50^{\circ}$, (4) $\theta=40^{\circ}$, (5) $\theta=30^{\circ}$.

ergy interval between $E_{p}$ and $E_{p}+d E_{p}$ is given by integration of (10) over the angles:

$$
\frac{d \sigma}{d E_{p}}=L_{0} \int_{0}^{\pi} W_{T}\left(E_{p}, \theta\right) d \sigma_{R}(\theta) .
$$

For different ion energies, $d \sigma / d E_{p}$ is shown for $\mathrm{U}+\mathrm{U}$ in Fig. 3(a). Compared with the purely spontaneous positron autoionization, ${ }^{6}$ the dynamical, nonadiabatic effects lead to a "smearing out" of the sharply peaked excitation functions and to a considerable increase of the cross section by nearly 2 orders of magnitude.

To get the total positron cross section $\sigma$, one has to integrate (11) over the positron energy $E_{p}$. As a function of the ion energy $E_{l}, \sigma$ is shown in Fig. 3(b). It is larger by nearly 2 orders of magnitude than the corresponding one for purely spontaneous positron autoionization. ${ }^{6}$ This feature is due to the induced transitions (nonadiabatic effects). In systems with higher total charge $Z_{1}+Z_{2}$, the transitions to higher levels $\left(2 p_{1 / 2}\right.$, $2 s_{1 / 2}$ ) which come close to diving or are just diving $\left(2 p_{1 / 2}\right)$ must be taken into account and will further increase the cross sections.

We acknowledge useful discussions with Dr. J. Rafelski, G. Soff, and Professor W. Scheid.

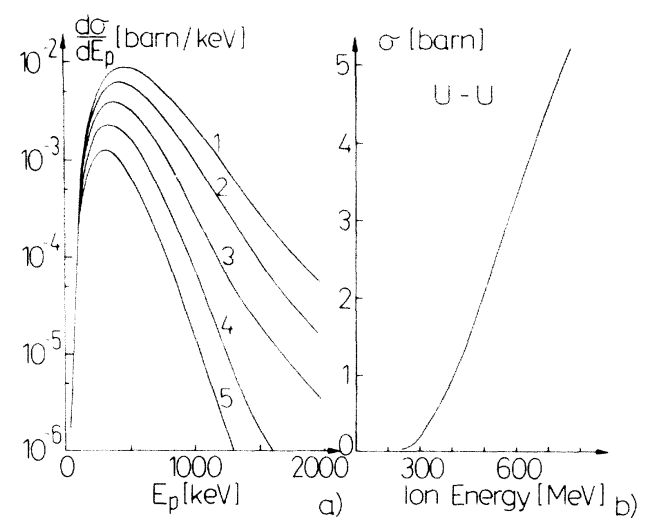

FIG. 3. (a) The positron production cross section $d \sigma /$ $d E_{p}$ for the system $U+U$ for various ion energies. The energies are the same as in Fig. 2(b). (b) The total positron cross section in dependence on the ion energy.

*Work supported by the Deutsche Forschungsgemeinschaft, by the Bundesministerium für Forschung und Technologie, and by the Gesellschaft für Schwerionenforschung (GSI).

†Work supported by the Alexander von Humboldt-Stiftung.

${ }^{1}$ The idea of intermediate superheavy molecules has first been mentioned by the authors in various GSI seminars, 1969-1970; see, e.g., K. Smith et al., in GSI-Bericht 72-7 (Gesellschaft für Schwerionenforschung, Darmstadt, Germany, 1972).

${ }^{2}$ J. Rafelski, L. P. Fulcher, and W. Greiner, Phys. Rev. Lett. 27, 958 (1971).

${ }^{3}$ B. Müller, H. Peitz, J. Rafelski, and W. Greiner, Phys. Rev. Lett. 28, 1235 (1972).

${ }^{4}$ B. Müller, J. Rafelski, and W. Greiner, Z. Phys. 257, 183 (1972); J. Rafelski, B. Müller, and W. Greiner, "The Charged Vacuum in Overcritical Fields" (to be published).

${ }^{5}$ P. H. Mokler, H. J. Stein, and P. Armbruster, Phys. Rev. Lett. 29, 827 (1972); W. E. Meyerhof, T. K. Saylor, S. M. Lazarus, W. A. Little, and B. B. Triplett, Phys. Rev. Lett. 30, 1279 (1973); F. W. Saris, W. F. van der Weg, H. Tawara, and W. A. Laubert, Phys. Rev. Lett. 28, 717 (1972); D. Burch, W. B. Ingalls, $\mathrm{H}$. Wieman, and R. Vandenbosch, " $K$-shell Ionization of $\mathrm{Pb}$ in $30-$ to $100-\mathrm{MeV} \mathrm{Cl}$ Collisions" (to be published); C. K. Davis, J. S. Greenberg, P. Vincent, and M. Yam, Bull. Amer. Phys. Soc. 18, 1406 (1973).

${ }^{6}$ H. Peitz, B. Müller, J. Rafelski, and W. Greiner, Lett. Nuovo Cimento $\underline{8}, 37$ (1973).

${ }^{7}$ K. Smith, B. Müller, and W. Greiner, "Dynamical Theory of Intermediate Molecular Phenomena in Heavy Ion Scattering" (to be published).

${ }^{8}$ B. Müller, J. Rafelski, and W. Greiner, Phys. Lett. 47B, 5 (1973). 